to occur. The cause of the disease must therefore be a deficiency in an antihomorrhagic factor different from vitamin $C$ and occurring in cereals and seeds.

The histological features of the symptoms as well as the concentration and further characterisation of the antihæmorrhagic factor are being investigated further.

Biochemical Institute,

H. DAM.

University,

Copenhagen.

May 2.

' H. Dam, Biochem. Z., 215, 485; 1929.
\& H. Dam, Biochem. Z., 220, $159 ; 1930$.

\section{Optical Rotatory Power}

AN interesting mathematical derivation of the rotatory power of a simple organic compound has just been published by S. F. Boys ${ }^{1}$, from which conclusions are drawn regarding, inter alia, the influence of solvents upon the magnitude of the rotation. The author's comments on the effects of association and his classification of active solutes into three main groups, namely, non-polar, polar and those capable of entering into co-ordination or complex union with the solvent, are in agreement with the views arrived at by one of us from experimental data ${ }^{2}$.

We are at present engaged in investigating the behaviour of optically active saturated hydrocarbons towards change of solvent, using $d$-pinane, methyl menthane and other compounds as the non-polar solutes. We find that the rotation in such cases is chiefly governed by the refractive index of the solvent medium. This point is illustrated by the following values for $d$-pinane in dilute solution :

$$
d \text {-Pinane in Solution }(c=3 \cdot 5) \text {. }
$$

\begin{tabular}{|c|c|c|c|c|c|}
\hline Solvent & $n_{D}$ & {$[\alpha]_{D}$} & Solvent & $n_{D}$ & {$[a] D$} \\
\hline$t$ & $1 \cdot 3460$ & $+18 \cdot 7^{\circ}$ & Methylene dichloride & $1 \cdot 4237$ & $-21 \cdot 0^{\circ}$ \\
\hline thyl a. & $1 \cdot 3312$ & $19 \cdot 3$ & Heptane & $1 \cdot 3867$ & 21 . \\
\hline cetic acid & $1 \cdot 3715$ & $19 \cdot 5$ & Chloroform & $1 \cdot 4464$ & $21 \cdot \varepsilon$ \\
\hline Nitromethane & $1 \cdot 3813$ & $19 \cdot 8$ & Carbon tetrachloride & 1.4607 & $22 \cdot 9$ \\
\hline Acetaldehyde & $1 \cdot 3316$ & $20 \cdot 1$ & Methyl iodide & 1.5293 & $23 \cdot 6$ \\
\hline Hexane & 1.3760 & $20 \cdot 1$ & Methylene iodide & $1 \cdot 7559$ & 26 \\
\hline Acetone & $1 \cdot 3589$ & $20 \cdot \overline{9}$ & Carbon disulphide & $1 \cdot 6204$ & 28 \\
\hline
\end{tabular}

A large number of aromatic solvents have also been examined, which fall into their appropriate positions in the above table. The refractive indices quoted are those of the pure solvent, but the results strongly support the prediction of S. F. Boys that the rotation of non-polar solutes will be dependent on the refractivity of the solution. An interesting point is that the specific rotation of pinane in the homogeneous state $\left(n_{D} 1 \cdot 4624,[\alpha] D+22 \cdot 95^{\circ}\right)$ is practically unaltered when the compound is dissolved in carbon tetrachloride (see table). In this case solvent and solute have almost identical refractive indices.

Similar results are being obtained with other hydrocarbons, full details of which will be published later.

\section{Department of Chemistry, University, Edinburgh. May 16.}

\section{A. R. Chambers.}

\section{H. Gordon Rule.}

${ }^{1}$ Proc. Roy. Soc., A, 144, 655, 675; 1934.

'Rule and co-workers, J. Chem. Soc., 384, 1217; 1933; and earlier papers.
Raman Spectra of Benzene and Hydrogen Iodide in the Liquid and Solid State

A systematic investigation at low temperatures of the oscillation and rotation Raman spectra of simple molecules as they are affected by their state of aggregation and by temperature seems to us of great importance; for the transition from the gaseous to the liquid state will show mainly the influence of density, while in the transition from the liquid to the solid state the orientation of the exchange forces due to the crystal structure may have some influence on the Raman lines. Moreover, having ascertained the dependence of the Raman spectra upon temperature, we are in the position to say something about the magnitude of the exchange forces and the rotation of the molecules in the crystal.

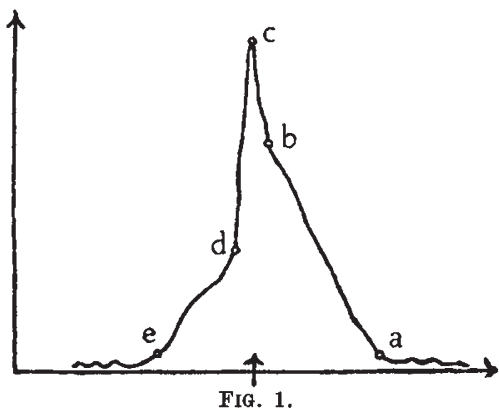

The whole investigation makes the utmost demands upon the low temperature apparatus as well as upon the spectroscopic arrangement. We have constructed a low temperature apparatus, which enables us to prepare a clear and transparent crystal and to keep it at a constant temperature $\left( \pm 0 \cdot 1^{\circ} \mathrm{C}\right.$. $)$ between $+30^{\circ}$ and $-150^{\circ} \mathrm{C}$. for any length of time (up to 100 hours) ; the type of spectrograph used is the big Steinheil with three glass prisms. In order to get the best possible results from it, we have increased the rigidity of its mechanical parts and have regulated the temperature of the room. As source of light we employed the line $\lambda 3888 \mathrm{~A}$. from a helium discharge tube to avoid any disturbance by a continuous background (for the details of the apparatus see the paper shortly to appear in Z. phys. Chem. (B)). The following are data for the spectrograph : dispersion at $\lambda 4200$ A., small camera $(f=270 \mathrm{~mm}$.) $21 \mathrm{~A} . / \mathrm{mm}$., large camera $(f=650 \mathrm{~mm}$.) $8 \cdot 7 \mathrm{~A} . / \mathrm{mm}$.; diameter of the camera lens $65 \mathrm{~mm}$.

We have obtained the following results :

Benzene.

\begin{tabular}{|c|c|c|c|c|}
\hline $\begin{array}{l}\left.m^{-1}\right) \text { for liquid } \\
m^{-1} \text { ) for solid }\end{array}$ & $\begin{array}{l}992 \cdot 2 \\
990 \cdot 5\end{array}$ & $\begin{array}{l}983 \cdot 3 \\
982 \cdot 3\end{array}$ & $\begin{array}{l}1176 \cdot 7 \\
1174 \cdot 7\end{array}$ & $\begin{array}{l}1605 \cdot 3 \\
1602 \cdot 9\end{array}$ \\
\hline $\begin{array}{l}\text { ecrease } \\
\text { ecrease }\end{array}$ & $\begin{array}{l}1.7 \\
1.7\end{array}$ & $\begin{array}{l}1 \cdot 0 \\
1 \cdot 0\end{array}$ & $\begin{array}{l}2 \cdot 0 \\
1 \cdot 7\end{array}$ & $2 \cdot 4$ \\
\hline
\end{tabular}

$\begin{array}{lcccc}\text { Decrease (per thousand) } & \ldots & 1.7 & 1.0 & 1.7\end{array}$ if we go from the liquid to the solid state. This change, which is much larger than the uncertainty of $\nu_{R}$ (not more than $0 \cdot 1-0 \cdot 3$ per thousand) means a small weakening of the binding forces of benzene in the solid state. The half-width of the line $992 \cdot 2 \mathrm{~cm} .^{-1}$ will be less than $4 \mathrm{~cm}^{-1}$, in accordance to Grassmann ${ }^{1}$.

Hydrogen Iodide.

\begin{tabular}{|c|c|c|}
\hline $\begin{array}{l}\text { Gaseous state } \\
\text { Liquid " }\end{array}$ & $\begin{array}{l}2233 \\
2164 \cdot 9 \pm 1 \cdot 0\end{array}$ & $\begin{array}{l}\mathrm{cm}^{-1} \\
\mathrm{~cm}^{-1}\end{array}$ \\
\hline $\begin{array}{l}\text { Decrease } \\
\text { Decrease per thousand } \\
\text { Solid state }\end{array}$ & $\begin{array}{l}\quad 68 \cdot 1 \\
31 \\
2159 \cdot 9 \pm 1 \cdot 0\end{array}$ & $\begin{array}{l}\mathrm{cm} .^{-1} \\
\mathrm{~cm} .^{-1}\end{array}$ \\
\hline $\begin{array}{l}\text { Decrease } \\
\text { Decrease per thousand }\end{array}$ & $\begin{array}{l}5 \cdot 0 \\
2 \cdot 3\end{array}$ & $\mathrm{~cm} .^{-1}$ \\
\hline
\end{tabular}

